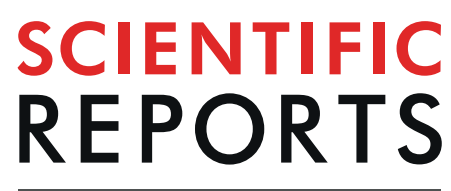

natureresearch

\title{
Structural properties of [2Fe-2S] ISCA2-IBA57: a complex of the mitochondrial iron-sulfur cluster assembly machinery
}

\author{
Veronica Nasta ${ }^{1,2,4}$, Stefano Da Vela ${ }^{3,4}$, Spyridon Gourdoupis ${ }^{1}$, Simone Ciofi-Baffoni ${ }^{1,2}$, \\ Dmitri I. Svergun ${ }^{3}$ \& Lucia Banci ${ }^{1,2 *}$
}

In mitochondria, a complex protein machinery is devoted to the maturation of iron-sulfur cluster proteins. Structural information on the last steps of the machinery, which involve ISCA1, ISCA2 and IBA57 proteins, needs to be acquired in order to define how these proteins cooperate each other. We report here the use of an integrative approach, utilizing information from small-angle X-ray scattering (SAXS) and bioinformatics-driven docking prediction, to determine a low-resolution structural model of the human mitochondrial [2Fe-2S] ${ }^{2+}$ ISCA2-IBA57 complex. In the applied experimental conditions, all the data converge to a structural organization of dimer of dimers for the [2Fe-2S $]^{2+}$ ISCA2-IBA57 complex with ISCA2 providing the homodimerization core interface. The [2Fe-2S] cluster is out of the ISCA2 core while being shared with IBA57 in the dimer. The specific interaction pattern identified from the dimeric [2Fe-2S] ${ }^{2+}$ ISCA2-IBA57 structural model allowed us to define the molecular grounds of the pathogenic Arg146Trp mutation of IBA57. This finding suggests that the dimeric [2Fe-2S] ISCA2-IBA57 hetero-complex is a physiologically relevant species playing a role in mitochondrial [4Fe-4S] protein biogenesis.

Iron-sulfur $(\mathrm{Fe}-\mathrm{S})$ clusters are among the most ancient inorganic protein co-factors ${ }^{1}$. The most common $\mathrm{Fe}-\mathrm{S}$ clusters found in nature are $[2 \mathrm{Fe}-2 \mathrm{~S}]$ and $[4 \mathrm{Fe}-4 \mathrm{~S}]$ clusters, which are formed by tetrahedrally coordinated iron atoms with bridging sulfides and are coordinated to the protein in the majority of cases through cysteine residues $^{2}$. These protein-bound clusters are essential players in a variety of biological processes ranging from electron transfer to enzymatic reactions ${ }^{3,4}$. Despite their simple chemical composition, the biosynthesis of Fe-S clusters is a complex and strictly regulated process involving multiple protein components ${ }^{4-6}$. In mitochondria, a complex protein machinery is devoted to the maturation of iron-sulfur cluster (ISC)-containing proteins and enzymes ${ }^{7,8}$. In a first step, this machinery, named mitochondrial ISC assembly machinery hereafter, assembles a [2Fe-2S] cluster $^{9}$. In a second step, the machinery combines two [2Fe-2S] clusters to assemble a [4Fe-4S] cluster, which is then inserted into mitochondrial target proteins $\mathrm{s}^{10,11}$. The molecular events at the basis of this second step are still quite elusive. Some of the members of this second step include the A-type ISC proteins, monothiol glutaredoxins and the IBA57 protein family. The human genome encodes, among the proteins localized in mitochondria, two A-type ISC proteins, termed ISCA1 and ISCA2, a monothiol glutaredoxin, GLRX5, and a IBA57 protein. GLRX5 works as a $[2 \mathrm{Fe}-2 \mathrm{~S}]$ cluster transfer protein in the mitochondrial matrix ${ }^{12,13}$. ISCAs and IBA57 proteins are required for the maturation of mitochondrial $[4 \mathrm{Fe}-4 \mathrm{~S}]$ proteins ${ }^{11,14,15}$, but their interaction network is still argument of debate in the literature. In S. cerevisiae it has been proposed that these proteins act in the same biochemical pathway, which encompasses a hetero complex formed by S. cerevisiae ISCA1, ISCA2, and IBA57 homologues as the functional unit devoted to convert two [2Fe-2S] clusters ${ }^{11,16}$, received by $S$. cerevisiae GLRX5 homologue ${ }^{13,17}$, to a $[4 \mathrm{Fe}-4 \mathrm{~S}]$ cluster $^{18,19}$. On the contrary, data collected on human cells suggested that a ternary complex among ISCA1, ISCA2, and IBA57 is not operative in the physiological state of human cells ${ }^{20}$. This model was based on the findings that, although ISCA1 and ISCA2 reciprocally interact, only ISCA2, and not ISCA1, was found

${ }^{1}$ Magnetic Resonance Center CERM, University of Florence, Via Luigi Sacconi 6, 50019, Sesto Fiorentino, Florence, Italy. ${ }^{2}$ Department of Chemistry, University of Florence, Via della Lastruccia 3, 50019, Sesto Fiorentino, Florence, Italy. ${ }^{3}$ European Molecular Biology Laboratory, Hamburg Outstation, EMBL c/o DESY, Notkestrasse 85, 22607, Hamburg, Germany. ${ }^{4}$ These authors contributed equally: Veronica Nasta and Stefano Da Vela. *email: banci@cerm.unifi.it 
to interact with IBA57, at variance with S. cerevisiae data ${ }^{20}$. Recently, we found that the three proteins GLRX5, ISCA 2 and IBA57 lead to the formation of a [2Fe-2S $]^{2+}$ ISCA2-IBA57 complex in vitro ([2Fe-2S] ISCA2-IBA57 hereafter $)^{21}$. Specifically, the latter complex is formed in two steps: first, the [2Fe-2S] cluster is transferred from [2Fe-2S] GLRX5 to apo ISCA2 and then IBA57 interacts with [2Fe-2S] ISCA2 forming the heterodimeric [2Fe2S] ISCA2-IBA57 complex. The latter complex was not formed with ISCA $1^{21}$, supporting the model that, in humans, ISCA proteins modulate their interactions network in a complex and dynamic manner. The [2Fe-2S] ISCA2-IBA57 complex is asymmetrically coordinated by the conserved cysteines Cys 79, Cys 144, and Cys 146 of ISCA 2 and by Cys 259 from IBA57, thus resulting the [2Fe-2S] cluster bridged between the two proteins ${ }^{21}$. We also showed that cluster binding is required to promote complex formation between ISCA2 and IBA57 proteins ${ }^{21}$, as indeed the apo proteins do not interact. Finally, each of the four cysteines are essential to induce the [2Fe-2S] hetero-complex formation, since, once individually mutated to Ala, the [2Fe-2S] ISCA2-IBA57 complex formation is abolished ${ }^{21}$. Overall, these data indicate that ISCA2, and not ISCA1, specifically interacts with IBA57 to form a cluster-mediated interaction.

Mutations in ISCA2 and IBA57 genes have been reported in patients affected by multiple mitochondrial dysfunctions syndromes (MMDS), specifically MMDS3 for IBA $57^{22-28}$ and MMDS4 for ISCA2 $2^{29,30}$. In these patients, the biochemical phenotype is similar and was characterized by a defect in the respiratory chain complexes I and II and a decrease in mitochondrial protein lipoylation, both effects resulting from impaired assembly of [4Fe-4S] clusters $^{25,26,31}$. It was also reported that the expression of ISCA2 is drastically decreased in IBA57 patients as well as ISCA2 patients have a decreased amount of IBA $57^{32}$, at support of a direct functional link of these two proteins ${ }^{26}$. All these data indicate, in agreement with the in vitro [2Fe-2S] ISCA2-IBA57 complex formation ${ }^{21}$, that ISCA2 and IBA57 operate in a synergistic way in the maturation of [4Fe-4S] proteins.

We report here the use of an integrative approach, utilizing information from small-angle X-ray scattering (SAXS) and bioinformatics-driven docking prediction to determine a low-resolution structural model of the human mitochondrial [2Fe-2S] ISCA2-IBA57 complex. Specifically, the SAXS data were combined with mutagenesis data and bioinformatic prediction-driven modelling to derive the model of the [2Fe-2S] ISCA2-IBA57 complex. Based on this structural model, we identified the specific [2Fe-2S] ISCA2-IBA57 interaction pattern, which allow us to rationalize the role of the pathogenic mutation Arg146Trp in IBA57.

\section{Results}

Structural information on IBA57 and apo ISCA2 by SAXS. The SAXS data on IBA57 suggested that the crystal structure (6QE3.pdb) ${ }^{21,33}$ is maintained in solution and the scattering computed from this structure fits well the experimental SAXS profile of IBA57 (discrepancy $\chi^{2}=1.075$, continuous line in Fig. 1a).

For the apo ISCA2 sample, the SAXS curve from the main chromatographic SEC-SAXS peak is not consistent with a monomeric $12.6 \mathrm{kDa}$ protein. Instead, the overall parameters presented in Table 1 point to the presence of an apo ISCA2 homodimer in solution, consistently with previous NMR data that showed a dimeric state for ISCA2 in both apo and holo forms ${ }^{18}$. To model the dimer, two different approaches were followed. In the first approach, the experimentally validated structure of the ISCA2 monomer obtained with Modeller (see Structural Coordinates paragraph of the Methods section) was used for rigid body modelling of the ISCA2 dimer assuming a P2 symmetry (see Methods). The best dimeric model (blue continuous line in Fig. 1b) gave a final $\chi 2$ of 1.063 against the SAXS curve of apo ISCA2 (green dots). In the second approach, ISCA2 dimers were generated by superimposing the Modeller monomer on existing crystal structures of homodimers of bacterial ISCA homologues (PDB: 2D2A, 1S98, 1R95). These dimeric structures were used to fit the SAXS curve of apo ISCA2 resulting in $\times 2$ values of 1.049 for 2D2A, 1.216 for $1 S 98$ and 1.136 for 1 R95 (Fig. 1b). All the dimer models obtained with the two approaches fitted well to the experimental SAXS patterns and revealed an overall low-resolution " 8 "-like shape (Fig. 1b, inset), but with different dimeric interfaces. Indeed, the two best models, with comparable $\chi 2$ values of 1.049 and 1.063, were obtained with structures having the dimeric interfaces differently exposing the cysteine ligands on the two subunits of the dimer. In the structure based on SufA bacterial homologue ( $\chi 2$ value of 1.049), the three cysteine ligands face each other from the two subunits of the dimer (Fig. 2a). On the contrary, in the rigid body modelling structure providing the $\chi 2$ value of 1.063 , the cysteine ligands of the two subunits are solvent exposed on the opposite side of the dimer (Fig. 2b). These two models yield very similar fits to the SAXS data (see Discussion).

Structural model of [2Fe-2S] ISCA2-IBA57 complex. A starting structural model of the [2Fe2S] ISCA2-IBA57 complex was first calculated by combining bioinformatics interface predictions with information-driven docking. This approach has already been applied with success to model protein-protein complexes whose interaction is metal-mediated ${ }^{34}$, a circumstance that applies to the formation of the [2Fe-2S] ISCA2-IBA57 complex ${ }^{21}$.

While the structure of IBA57 is available ${ }^{21,33}$, the structure of human [2Fe-2S]-bound ISCA2 is not. However, a [2Fe-2S] cluster-bound crystal structure of IscA from Thermosynechococcus elongatus has been solved ${ }^{35}$. In this structure, one partially exposed [2Fe-2S] cluster is coordinated by two conformationally distinct IscA molecules. An asymmetric cysteinyl ligation occurs via the conserved cysteines Cys 37, Cys 101, Cys 103 from one molecule and Cys 103 from the other, with the latter molecule providing only one cysteine ligand to cluster coordination. This cysteine has been shown to have more conformational flexibility than the other coordinating cysteines provided by the other protein molecule ${ }^{35}$. Considering that we have previously showed that ISCA 2 coordinates the cluster in the [2Fe-2S] ISCA2-IBA57 complex with the same three Cys as found in the first molecule of the bacterial dimer ${ }^{21}$, we modelled human ISCA2 sequence on this molecule of IscA from T. elongates. The obtained structural model of human ISCA2 has a cluster coordination where an iron ion is coordinated by Cys 144 and Cys 146 and the other iron ion is coordinated by Cys 79 ([2Fe-2S] ISCA2, hereafter). The vacant coordination site of the latter iron ion is occupied in the complex by Cys 259 of IBA57 (corresponding to Cys 230 in the produced protein 

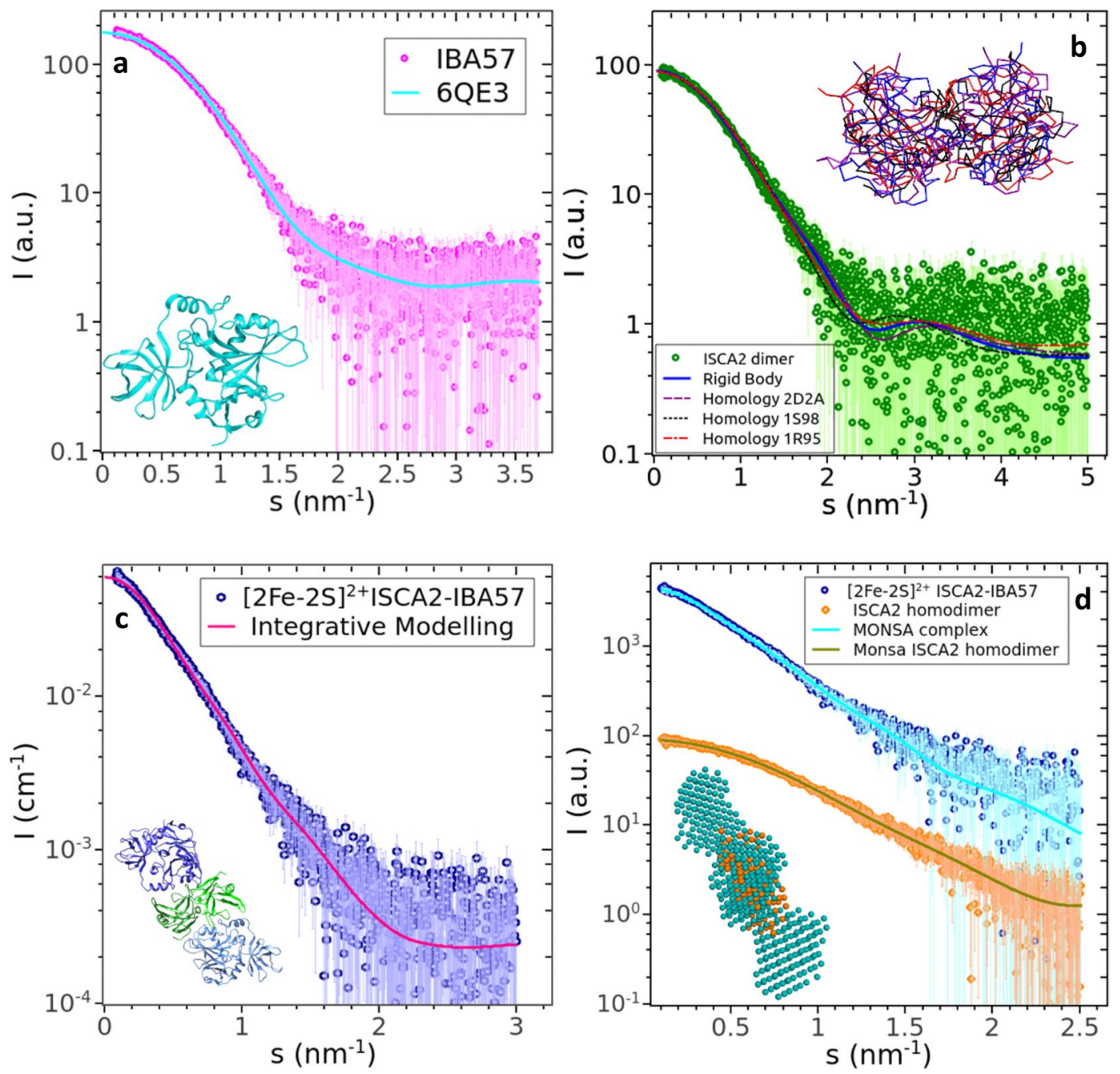

Figure 1. SAXS curves and curve fitting of IBA57, apo ISCA2 and [2Fe-2S] $]^{2+}$ ISCA2-IBA57. (a) SAXS profile of IBA57 and the fit computed from the crystal structure 6QE3 (ribbon diagram in the inset). (b) SAXS profile of ISCA 2 homodimer and fits by rigid body and homology models. Inset: backbones of the fitted dimers showing similar overall architecture, superimposed with SUPCOMB ${ }^{67}$, with the same color-coding as the fit curves. (c) Rigid body fit using cluster 1 heterodimers. Inset: the model of the complex in which dimerization is mediated by ISCA2. (d) Simultaneous multiphase ab initio fit to the complex and to the ISCA2 homodimer. Inset: twophase model; orange beads, ISCA2 homodimer, teal beads, the complex. The scattering intensity is displayed as a function of the momentum transfer $s=4 \pi \sin (\theta) / \lambda$, where $2 \theta$ is the scattering angle and $\lambda$ is the X-ray wavelength.

construct lacking the mitochondrial targeting sequence of 30 amino acids), since the latter Cys is required to form the cluster-mediated [2Fe-2S] ISCA2-IBA57 hetero-complex (as it occurs with Cys 79, Cys 144, Cys 146 of ISCA2 $)^{21}$. The IBA57 and [2Fe-2S] ISCA2 structures were employed as input to obtain the [2Fe-2S] ISCA2-IBA57 complex using a data-driven docking HADDOCK approach ${ }^{36,37}$. The latter approach requires ambiguous interaction restraints (AIRs) to successfully drive the protein-protein docking. To obtain AIRs, we used a bioinformatics approach that was already used by us in predicting protein-protein interfaces of metal-driven protein-protein complexes $^{34}$. Briefly, the interface prediction program WHISCY ${ }^{38}$ was used to obtain the residues predicted to be involved in the protein-protein interface of the [2Fe-2S] ISCA2-IBA57 complex (Supplementary Table S1 and Fig. 3) and directly extracting the consequent AIRs provided by the program as an output. In the HADDOCK calculations, unambiguous interaction restraints between the iron ions and the iron coordinating cysteines of both proteins were also used (for details see Methods). The calculated models have been clustered on the basis of common contacts following standard HADDOCK scoring approach ${ }^{39}$ (Supplementary Table S2, for details see Methods). The obtained clusters of structural models can be grouped in two families ( $a$ and $b)$ on the basis of the RMSD among the various clusters using a threshold of $\sim 2 \AA$ (Fig. 4). The backbone RMSD values of $a$ and $b$ families are 1.01 and $1.28 \AA$, respectively, (Supplementary Fig. S1); the two cluster families differ of $\sim 10 \AA$ (Fig. 4), as a consequence of $\mathrm{a} \sim 180^{\circ}$ rotation around $\mathrm{z}$-axis of one protein to the other in the complex, as it can be observed by comparing the best model of each family (Supplementary Fig. S2). In both families, the [2Fe-2S] cluster is buried at the interface of the two proteins (Fig. 5). In the overall best scoring model of family $a$, protein-protein 


\begin{tabular}{|c|c|c|c|}
\hline Data collection parameters & $\begin{array}{l}\text { Robotic sample } \\
\text { changer }\end{array}$ & \multicolumn{2}{|l|}{ SEC-SAXS } \\
\hline Radiation source & PETRA III (DESY) & \multicolumn{2}{|c|}{ PETRA III (DESY) } \\
\hline Beamline & EMBL P12 & \multicolumn{2}{|l|}{ EMBL P12 } \\
\hline Detector & Pilatus 6M & \multicolumn{2}{|l|}{ Pilatus 6M } \\
\hline Wavelength & $0.124 \mathrm{~nm}$ & \multicolumn{2}{|l|}{$0.124 \mathrm{~nm}$} \\
\hline Sample-to-detector distance & $3.1 \mathrm{~m}$ & \multicolumn{2}{|l|}{$3.1 \mathrm{~m}$} \\
\hline$s$ range & $0.017-7.3 \mathrm{~nm}^{-1}$ & \multicolumn{2}{|c|}{$0.026-7.3 \mathrm{~nm}^{-1}$} \\
\hline Exposure time & $40 \times 0.1 \mathrm{~s}$ & \multicolumn{2}{|l|}{$1 \mathrm{~s} /$ frame } \\
\hline Temperature & $293.2 \mathrm{~K}$ & \multicolumn{2}{|l|}{$293.2 \mathrm{~K}$} \\
\hline Parameter (unit) & IBA57 & $(\text { ISCA2 })_{2}$ & $(\text { ISCA2-IBA57 })_{2}$ \\
\hline $\mathrm{Rg}^{\mathrm{a}}$ Guinier (nm) & $2.16 \pm 0.02$ & $2.03 \pm 0.05$ & $3.95 \pm 0.12$ \\
\hline $\operatorname{Rg} \operatorname{PDDF}^{b}(\mathrm{~nm})$ & 2.17 & 2.07 & 4.03 \\
\hline $\operatorname{Dmax}^{c}(n m)$ & $7.1 \pm 0.1$ & $6.7 \pm 0.1$ & 12.4 \\
\hline $\mathrm{Vp}^{\mathrm{d}}\left(\mathrm{nm}^{3}\right)$ & $66 \pm 10$ & $25 \pm 10$ & $168 \pm 10$ \\
\hline $\operatorname{MrI}(0)$ & $\mathrm{n} / \mathrm{a}$ & $\mathrm{n} / \mathrm{a}$ & 79900 \\
\hline $\mathrm{Mr}^{\mathrm{e}}($ from $\mathrm{Vp})$ & $41300 \pm 8260$ & $15400 \pm 3080$ & $105000 \pm 21000$ \\
\hline Mr Bayes $^{66}$ & $25250-29250$ & $17750-20250$ & $61600-75300$ \\
\hline Mr sequence & 34925 & 25130 & 101660 \\
\hline
\end{tabular}

Table 1. Overall parameters from the SAXS profiles used for the modeling. ${ }^{a}$ Rg: radius of gyration. ${ }^{b} \mathrm{PDDF}$ pair distance distribution function. ${ }^{\mathrm{c} D m a x}$ : maximal intramolecular distance. ${ }^{\mathrm{d}} \mathrm{Vp}$ : particle volume. ${ }^{\mathrm{e}} \mathrm{Mr}$ relative molar mass.

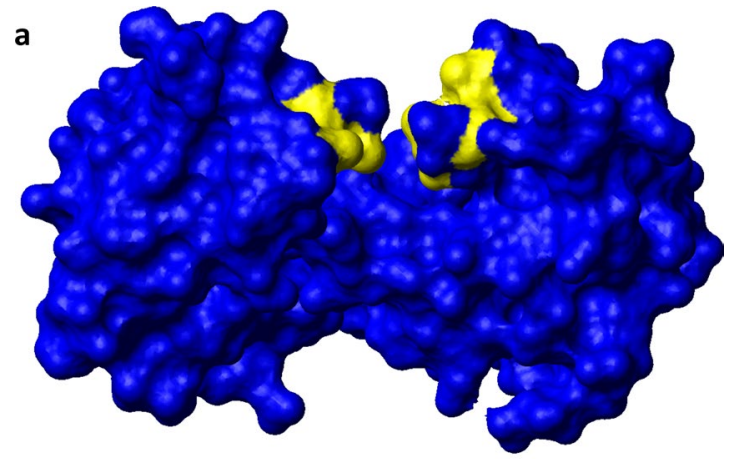

b

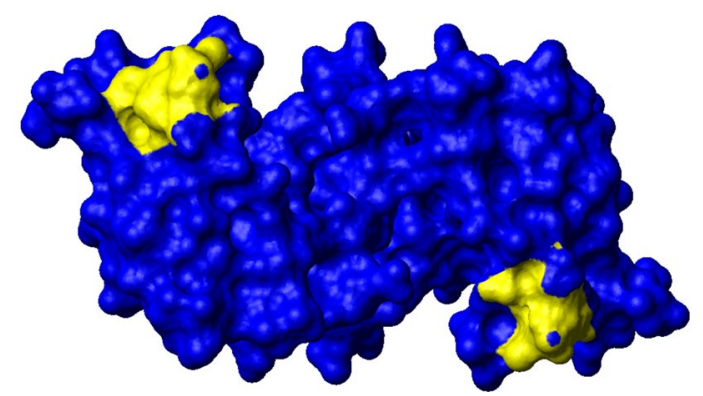

Figure 2. Structural models of apo ISCA2. Contact surface of dimeric apo ISCA2 obtained by the crystal structure of homodimeric SufA (PDB ID: 2D2A) (a) and by rigid body modelling assuming P2 symmetry (b). The yellow regions identify the Cys ligands.

recognition is stabilized by two intermolecular electrostatic interactions (Asp 111 (ISCA2) with Arg 146 (IBA57) and Glu 126 (ISCA2) with Arg 268 (IBA57)) which surround the cluster on opposite sides and by few hydrophobic interactions involving four residues at the protein-protein interface (Fig. 5a). The interaction involves a relatively small surface of $\sim 1400 \AA^{2}$, a value typically found in metal-mediated protein-protein interactions ${ }^{40,41}$. In family $b$, electrostatic and hydrophobic contacts as well as the buried surface area value are similar to those of family $a$ (Fig. 5b), thus explaining why both structural arrangements are similarly favoured (Supplementary Table S2). Indeed, comparing the two families, the same two arginine residues of IBA57 (Arg 146 and Arg 268) are involved in electrostatic interactions with Glu 126 (in family $b$ ) or Asp 111 (in family $a$ ) and with Glu 75 (in 


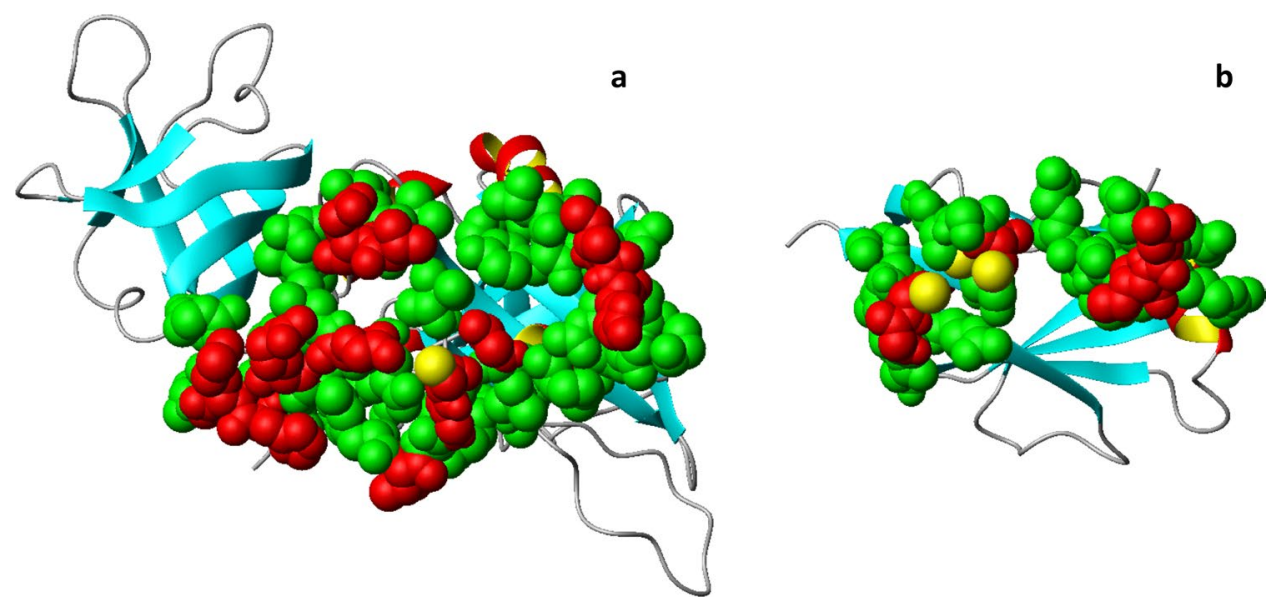

Figure 3. Predicted interacting residues of IBA57 and ISCA 2 mapped on the respective structures, obtained by the interface prediction program WHISCY and by the protein-protein docking program HADDOCK. Space filling representation of active (red) and passive (green) residues used for docking (see Supplementary Table S1 for the corresponding residue numbers) on the ribbon diagram of IBA57 (a) and ISCA2 (b). Active residues are defined based on interface predictions using WHISCY, passive residues are surface neighbors of active residues defined by HADDOCK (see Methods). Yellow spheres indicate the S $\gamma$ of Cys ligands, i.e. Cys 259 in IBA57 and Cys 79, Cys 144 and Cys 146 in ISCA2.

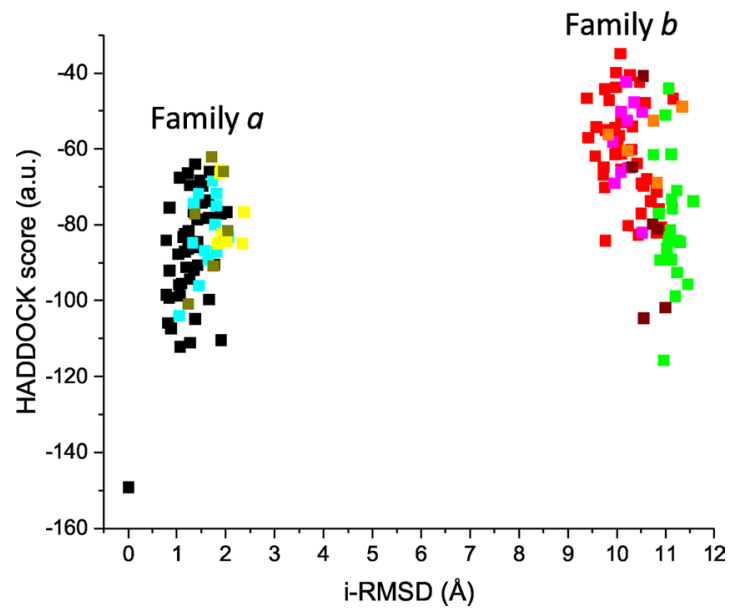

Figure 4. Clustering the models calculated by HADDOCK on the basis of common contacts. The plot is based on water-refined models generated by HADDOCK runs. The clusters (indicated with different colors in the plot) are based on the interface-ligand RMSDs calculated by HADDOCK, with the interface defined automatically based on all observed contacts. The clusters result and grouped in two families, $a$ and $b$. i-RMSD indicates the interface-RMSD calculated on the backbone (CA, C, N, O) atoms of all residues involved in intermolecular contact using a $10 \AA$ cutoff. This structural analysis was made with respect to the best HADDOCK model (the one with the lowest HADDOCK score).

family $b$ ) or Glu 126 (in family $a$ ) of ISCA2, respectively (Fig. 5 and Supplementary Fig. S1), and a similar hydrophobic patch involving the same ISCA2 residues in both structural models, i.e. Leu 127 and Ile 128 are present at the protein-protein interface of both families (Fig. 5). In particular, the electrostatic interaction involving Arg 146 of IBA 57 shows that the average distance between the two oppositely charged residues is significantly shorter in family $b$ than in family $a$, providing a potential criterion for discriminating between the two families of structures. In conclusion, two structural models for the [2Fe-2S] ISCA2-IBA57 complex with comparable HADDOCK scores were obtained through the bioinformatic prediction-driven modelling approach.

SAXS data collected on the [2Fe-2S] ISCA2-IBA57 complex (Supplementary Fig. S3) showed essentially no concentration effect. The overall parameters calculated from the SAXS curve (Table 1) clearly pointed to an oligomerization state larger than a heterodimer in these experimental conditions, and indeed a rigid-body modelling assuming a heterodimer did not satisfactorily fit the data. The two best scoring dimer models from family $a$ and $b$ were thus used for the rigid-body modelling against the SAXS curve of the [2Fe-2S] ISCA2-IBA57 complex assuming a dimer of dimers (see Methods). The resulting prolate dimers of dimers are similar in the overall shape for both dimeric models from family $a$ and $b$, and fit the data with similar quality (final $\times 21.314-2.118$ for 

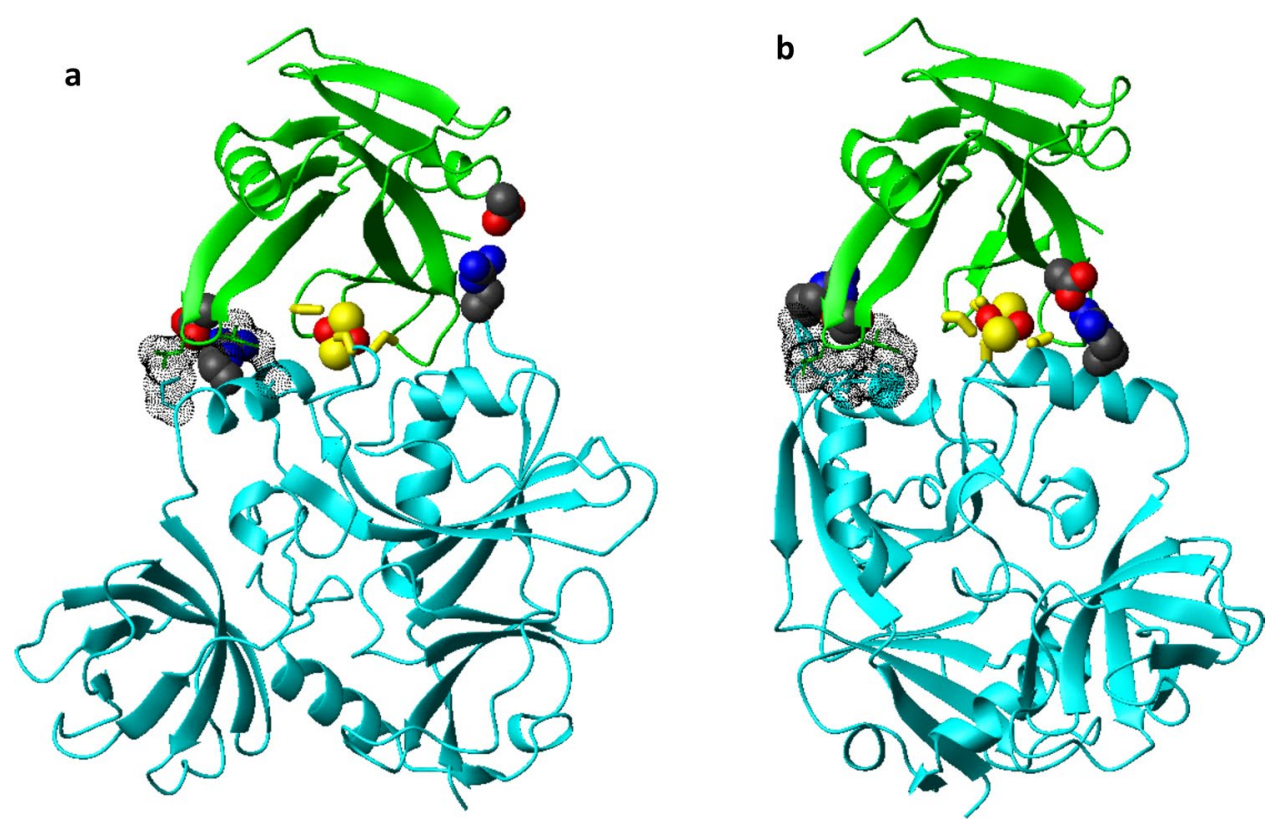

Figure 5. Structural models of the heterodimeric [2Fe-2S] ISCA2-IBA57 complex obtained by HADDOCK. Ribbon diagram of the best scoring HADDOCK model of [2Fe-2S] ISCA2-IBA57 in family $a$ (from cluster 1) (a) and in family $b$ (from cluster 3) (b). IBA57 is in cyan, ISCA2 is in green. The Cys ligands are shown as yellow stick, the iron and sulfur atoms of the [2Fe-2S] cluster are in red and yellow spheres, respectively. Intermolecular electrostatic interactions between Asp 111 (ISCA2) and Arg 146 (IBA57) and between Glu 126 (ISCA2) and Arg 268 (IBA57) in (a), and between Glu 75 (ISCA2) and Arg 268 (IBA57) and between Glu 126 (ISCA2) and Arg 146 (IBA57) in (b) are shown in CPK mode. Hydrophobic contacts between Ile 128 (ISCA2) and Ala 267 (IBA57) and between Leu 127 (ISCA2) and Met 272 (IBA57) in (a), and between Leu 127 (ISCA2), Ile 128 (ISCA2), Leu 142 (IBA57), Tyr 143 (IBA57), Ile 145 (IBA57) in (b) are shown in dots. The side-chains of the latter residues are shown as sticks.

family $a 1$ and 1.332-2.268 for family $b$ ). A subset of the solutions features an ISCA2 homodimer core (Fig. 1c $\chi 2=1.407)$. Interestingly, the core ISCA2 dimer extracted from this model also fits reasonably well the SAXS profile of the isolated ISCA2 homodimer, as displayed in Supplementary Fig. S4. The arrangement of ISCA2 at the core of the dimer of dimers with respect to IBA57 is somewhat less compact for the rigid body models obtained employing the best scoring heterodimer from family $b$ than that from family $a$ (Supplementary Fig. S5). Another subset of rigid body models, both for family $a$ and $b$, feature the ISCA2 molecules at the periphery, with IBA57 providing the homodimerization interface (Supplementary Fig. S6). Also these models are also in agreement with the SAXS data collected on the complex. Moreover, the orientation and the dimerization interface of ISCA2 may of course differ in the apo ISCA2 homodimer and in the dimer of [2Fe-2S] ISCA2-IBA57 heterodimers. On the other hand, performing ab initio multiphase modelling (see Methods) for a prolate dimer of dimers fitting simultaneously the SAXS curves from the ISCA2 homodimer and from the complex, it results that the obtained model features ISCA2 at the core of the assembly (Fig. 1d, $\chi 2=1.197$ for the curve of the complex and 0.961 for the curve of the ISCA2 homodimer). Thus, $a b$ initio modelling indicates that the formation of the dimer of dimers assembly via ISCA2 can be considered more probable than the dimer of dimers assembly via IBA57.

Molecular grounds of the pathogenic impact of the aminoacid exchange Arg146Trp in IBA57. Some of the WHISCY-identified residues of IBA57 (Arg105Trp, Arg146Trp, Val253Leu, Ile261Thr) and ISCA2 (Gly77Ser) located at or around the protein-protein interface in the complex, are mutated in patients affected by multiple mitochondrial dysfunction syndromes ${ }^{22-28,42,43}$. Among them, Arg 146 is the most solvent-exposed and is located close to Cys 259 ligand, suggesting that it can play a role in the [2Fe-2S] ISCA2-IBA57 complex formation. The pathogenic mutation of Arg 146 to Trp does not significantly affect the stability of IBA57 in vivo ${ }^{22}$, at variance with what previously described in other pathogenic missense mutation $\mathrm{s}^{26-28}$, but however impairs its function. All together, these data strongly suggest that the Arg 146 mutation affects IBA57 function because of its altered interaction with ISCA2. We have therefore investigated such hypothesis, by producing the Arg146Trp IBA57 mutant and testing its interaction with ISCA2. We found that the two proteins do not interact with each other in their apo form similar to what found for the wild-type proteins. Upon chemical reconstitution of a 1:1 Arg146Trp IBA57/ apo ISCA2 mixture with Fe-S cluster, no hetero-complex was formed. Indeed, the UV-vis spectrum recorded on the latter mixture compares well with that of [2Fe-2S] ISCA2 and not with that of the chemically reconstituted [2Fe-2S] ISCA2-IBA57 complex. Specifically, the band at $540 \mathrm{~nm}$, which is a marker of the formation of the [2Fe-2S] ISCA2-IBA57 hetero-complex ${ }^{21}$, is not present in the UV-vis spectrum of the chemically reconstituted ISCA2-Arg146Trp IBA57 mixture (Fig. 6a). Consistent with no complex formation, the analytical gel filtration of the latter mixture did not show the presence of any peak at elution volumes higher than those of monomeric 

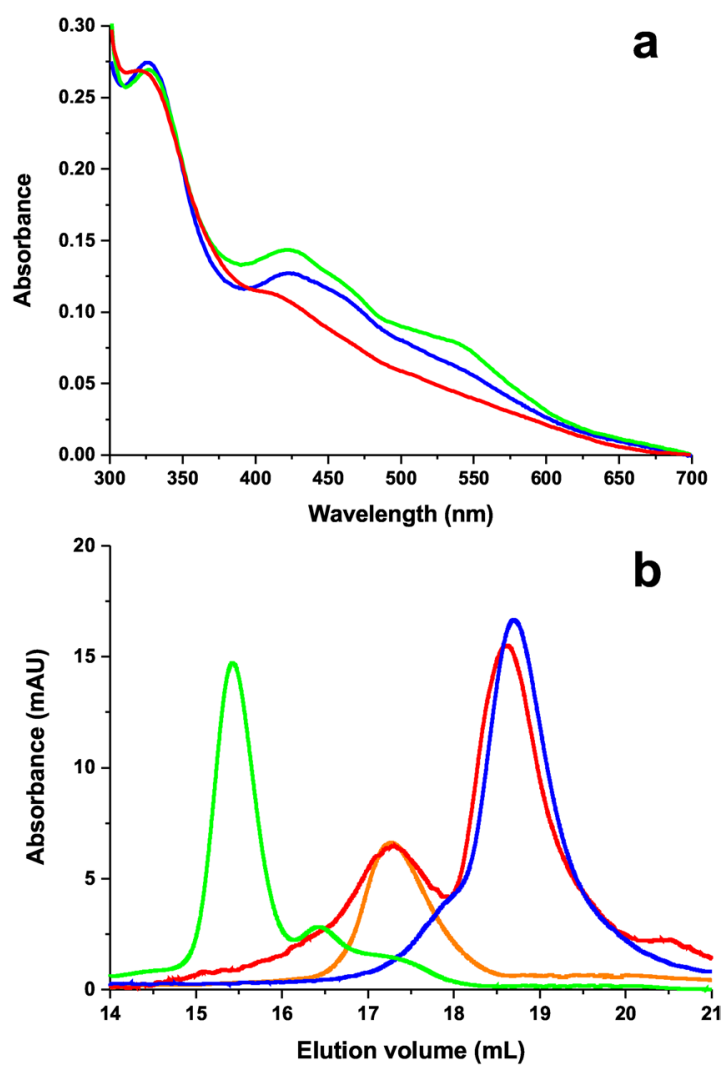

Figure 6. The pathogenic Arg146Trp mutation in IBA57 abolishes complex formation with ISCA2. (a) UVvisible spectra of the Fe-S chemically reconstituted 1:1 Arg146Trp IBA57/apo ISCA2 mixture (red), [2Fe2S] ISCA2 (blue) and the [2Fe-2S] ISCA2-IBA57 hetero-complex (green). (b) Elution profiles obtained for Arg146Trp IBA57 (blue), dimeric apo ISCA2 (orange), the [2Fe-2S] ISCA2-IBA57 hetero-complex (green) and a Fe-S chemically reconstituted 1:1 mixture of Arg146Trp IBA57 and apo ISCA2 (red), obtained by gel filtration on analytical Superdex 200 Increase 10/300 GL column. mAU: milli absorbance unit.

Arg146Trp IBA57 and dimeric apo ISCA2 as well as a peak at the elution volume of the wild-type hetero complex is not present (Fig. 6b). This result is in agreement with the HADDOCK structural models (families $a$ and $b$ ) which showed, indeed, that Arg 146 is involved in an electrostatic interaction at the protein-protein interface (Fig. 5). In particular, the observation that this electrostatic interaction is stronger in family $b$ than in family $a$ (see above) and that its disruption by Arg146 mutation abolishes complex formation suggests that family $b$ is the favorite structural organization of the [2Fe-2S] ISCA2-IBA57 hetero-complex. In conclusion, the pathogenic effects of the Arg146Trp mutation ${ }^{22}$ are likely a consequence of the impairment of the complex formation between IBA57 and ISCA2. This conclusion supports the proposal that the [2Fe-2S] ISCA2-IBA57 hetero-complex is a physiologically relevant species playing a role in mitochondrial $[4 \mathrm{Fe}-4 \mathrm{~S}]$ protein biogenesis.

\section{Discussion}

Several in vivo and in vitro data strictly associate human IBA57 and ISCA2 as proteins working in late steps of the ISC assembly machinery. Patients with pathogenic IBA57 mutations, that markedly reduce the protein levels of IBA57 in fibroblasts mitochondria, displayed expression levels of ISCA2 drastically decreased in all the patients ${ }^{26}$, as well as ISCA2 patients have a decreased amount of IBA $57^{32}$. Proteomic studies indicated that ISCA2 specifically interacts with IBA57 and not with ISCA $1^{20}$. This specific ISCA2-IBA57 interaction is helpful to rationalize the effects that the pathogenic mutations in IBA57 and ISCA2 have, i.e. the reduced protein levels of ISCA2 and IBA57 respectively induced by IBA57 and ISCA2 mutations can be interpreted as a lack of hetero-complex formation. Finally, we have recently described an in vitro [2Fe-2S] cluster transfer pathway involving ISCA2, IBA57 and GLRX5, which leads to the formation of a [2Fe-2S] ISCA2-IBA57 complex, which is resistant to highly oxidative environments and is capable of reactivating apo aconitase $\mathrm{e}^{21}$.

To gain structural insights on the stringent functional association characterizing IBA57 and ISCA2 proteins, an integrative approach utilizing information from SAXS and bioinformatics-driven docking prediction was used to determine a low-resolution structural model of the human mitochondrial [2Fe-2S] ISCA2-IBA57 complex. SAXS data on IBA57 and apo ISCA2 showed that the two proteins are, respectively, monomeric and dimeric in solution, in agreement with previous studies ${ }^{18,21}$. The SAXS data are in agreement with the solved crystal structure of IBA $57^{21,33}$, indicating that this structural arrangement is preserved in solution. Since no structure is available for dimeric apo ISCA2, structural models were obtained either by SAXS-guided rigid-body modeling of the dimer of ISCA2 from monomeric ISCA2 structural model or by imposing the same dimerization as in the crystal 


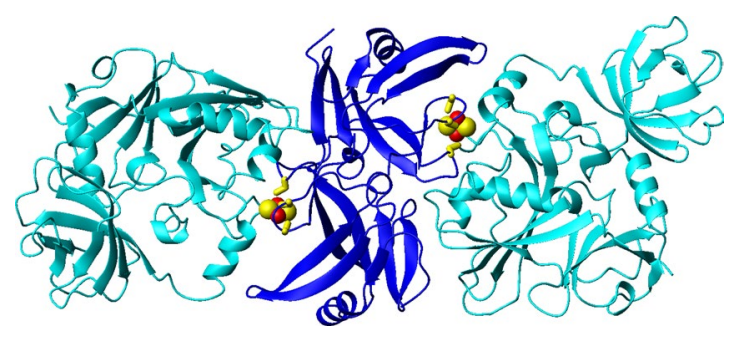

Figure 7. Structure of the dimer of dimers of [2Fe-2S] ISCA2-IBA57. The structure was obtained by rigidbody modelling of the [2Fe-2S] ISCA2-IBA57 complex against the SAXS curve of the [2Fe-2S] ISCA2-IBA57 complex using the best scoring dimeric model of family $a$. IBA57 and ISCA2 proteins are in cyano and blue, respectively. [2Fe-2S] clusters and Cys ligands are shown in CPK and stick modes, respectively.

structures of bacterial ISCA homologues. These models were used to interpret the SAXS data of apo ISCA2. All the fits led to two modes of ISCA2 dimerization, both sharing a "8"-like shape but with different dimeric interfaces (solvent exposed $v$ s. facing cysteines, Fig. 2a,b), and with fits of similar quality to the experimental SAXS profiles, thus preventing a conclusive definition of the precise orientation of the ISCA2 subunits in the dimer. On the other hand, apo ISCA proteins are known to assume different dimerization modes as observed in bacterial ISCA protein structures, such as that of the crystal structures of $E$. coli IscA $^{44,45}$. Thus, assuming that the two dimerization modes (solvent exposed $v s$. facing cysteines, Fig. 2a,b) are both possible in human apo ISCA2 as it occurs for bacterial apo ISCAs, we may suggest that the "facing cysteines", SufA-like, dimer is formed to allow the binding of the Fe-S cluster at the subunit-subunit interface of the ISCA2 homodimer. This is, indeed, supported by the fact that it has been previously proposed, on the basis of NMR data ${ }^{18}$, that apo ISCA2 dimerizes through the same inter-subunit contact region observed in SufA. The apo ISCA2 dimer, which exposes the cysteine of the two subunits on the opposite sides is, on the contrary, a structural arrangement suitable for sharing the Fe-S cluster, coordinated by three ISCA 2 cysteines, with a partner protein which then donates the fourth cysteine ligand, as it occurs in the ISCA2-IBA57 complex. We have recently proposed that reductive coupling of two, GLRX5-donated, $[2 \mathrm{Fe}-2 \mathrm{~S}]^{2+}$ clusters to form a single $[4 \mathrm{Fe}-4 \mathrm{~S}]^{2+}$ cluster on homodimeric ISCA2, requires six Cys ligands (three for each ISCA molecule) facing each other to promote the [4Fe-4S] cluster formation ${ }^{19}$. On this basis, we can assume that the facing cysteines structural model of ISCA2 dimer (Fig. 2b) and possibly also of the ISCA1-ISCA2 dimer ${ }^{18,46}$ is able to assemble a [4Fe-4S] cluster, while the ISCA2-IBA57 complex with only four cysteines available cannot assemble a [ $4 \mathrm{Fe}-4 \mathrm{~S}]$ cluster on its own by receiving two [2Fe-2S] clusters form GLRX5, but can only receive from GLRX 5 and binds a $[2 \mathrm{Fe}-2 \mathrm{~S}]$ cluster $^{21}$. On the other hand, considering i) the genomic data correlating IBA57 protein family to oxidative stress ${ }^{47,48}$, ii) the stability of the $[2 \mathrm{Fe}-2 \mathrm{~S}]$ cluster against oxidative degradation, when bound to the ISCA2-IBA57 complex with respect to that bound to ISCA2 alone ${ }^{21}$, and iii) the ability of the [2Fe-2S] ISCA2-IBA57 complex to reactivate in vitro the oxidatively damaged [4Fe-4S] aconitase $^{21}$, we can suggest that the [2Fe-2S] ISCA2-IBA57 heterocomplex can play a role in forming/repairing [4Fe-4S] clusters under aerobic cellular conditions by transferring one/two [2Fe-2S] clusters to mitochondrial target proteins which directly generate the $[4 \mathrm{Fe}-4 \mathrm{~S}]$ cluster. In conclusion, it might be possible that two alternative [4Fe-4S] assembly pathways, i.e. ISCA1-ISCA2- and IBA57-ISCA2-related pathways, are operative upon specific cellular conditions. The possible presence of the two alternative pathways is in agreement with the strict functional association found between ISCA 1 and ISCA 2 and between ISCA 2 and IBA57, but not between ISCA1 and IBA57. Indeed, both in vivo and in vitro data showed that ISCA1 and ISCA2 interact each other and only ISCA2, and not ISCA1, interacts with IBA $57^{18,21,46}$. Moreover, ISCA2 and IBA57 pathogenic mutations markedly reduce the protein levels of IBA57 and ISCA2, respectively, in the patients ${ }^{26,32}$, while the protein levels of ISCA1 are not affected or even slightly overexpressed in the patients affected by IBA57 pathogenic mutations ${ }^{26}$. The latter effect is in agreement with studies in HeLa cells where silencing of IBA57 was associated to unchanged or increased expression levels of ISCA $1^{14}$.

The SAXS data on the [2Fe-2S] ISCA2-IBA57 heterocomplex point to an oligomerization state larger than a heterodimer, being nicely modelled $a b$ initio by a dimer of dimers with prolate anisometry and featuring density for the ISCA2 homodimer at its core. The WHISCY-HADDOCK derived structural models of the heterodimeric [2Fe-2S] ISCA2-IBA57 complex (family $a$ and family $b$ ) well fit with the SAXS data, with a subset of solutions featuring the ISCA2 homodimer at its core, in agreement with the ab initio modelling. Furthermore, the ISCA2 homodimer core taken from the rigid-body modelling of the complex (obtained by family $a$ model) also fits the SAXS profile of the ISCA2 homodimer. We can thus reasonably propose that the dimeric ISCA2 in the complex exposes the three cysteine ligands on opposite sides in order to share the Fe-S cluster with IBA57, i.e. in a structural arrangement reproducing the "solvent exposed cysteines" dimer of apo ISCA2. The structure of the dimer of dimers of the [2Fe-2S] ISCA2-IBA57 complex, obtained by rigid-body modelling the SAXS data of the complex with family $a$ model, shows that the [2Fe-2S] cluster is, indeed, out of the ISCA2 core and shared with IBA57 (Fig. 7). We regard this assembly as the most consistent with all the available experimental data.

The dimer of dimers structural organization found in the [2Fe-2S] ISCA2-IBA57 differs from what we previously observed by running SEC-MALS on the [2Fe-2S] ISCA2-IBA57 complex ${ }^{21}$. The latter elutes in SEC-MALS with a molar mass of $51.2 \pm 0.5 \mathrm{kDa}$, corresponding to a heterodimeric complex formed by one monomeric ISCA2 (12565 Da) and one monomeric IBA57 (34925 Da). This different behaviour can be rationalized considering the different experimental conditions used to obtain SEC-MALS vs. SAXS data, i.e. the on-line size-exclusion 
chromatography was indeed avoided in order to reach the protein concentration required to run high quality SAXS data of the complex. Thus, it appears that both protein concentration and size-exclusion chromatography conditions affect the oligomerization state of the [2Fe-2S] ISCA2-IBA57 complex. Consistently, more and more NMR signals are observed in ${ }^{1} \mathrm{H}-{ }^{15} \mathrm{~N}$ HSQC spectra acquired on [2Fe-2S] ${ }^{15} \mathrm{~N}$-labelled ISCA2-unlabelled IBA57 upon decreasing complex concentrations (Supplementary Fig. S7). This suggests the presence of an equilibrium in solution between higher and lower molecular weight forms. The dimer of dimers is favoured at high concentrations of the complex and its signals are largely undetectable in the ${ }^{1} \mathrm{H}-{ }^{15} \mathrm{~N}$ HSQC spectrum, while the heterodimeric complex is favoured at low complex concentrations, whose signals became detectable in the ${ }^{1} \mathrm{H}_{-}{ }^{15} \mathrm{~N}$ HSQC spectrum. Therefore, all these data indicated that the [2Fe-2S] ISCA2-IBA57 complex is preferentially present in diluted solutions as heterodimer and that the latter has a high tendency to dimerize via the interaction of two ISCA2 molecules of two heterodimers. These results support the proposal that the dimer of dimers is induced by the in vitro conditions used in SAXS measurements and that the heterodimeric species is most likely the physiologically relevant species. In support to this model, we found that the WHISCY approach applied to define the ISCA2-IBA57 interaction identified on ISCA2 only the interaction surface involving the conserved cysteine cluster binding ligands, i.e. that surface interacting with IBA57. No other interaction regions were predicted by WHISCY. Therefore, WHISCY approach does not provide any clues on the residues at the interface of the two ISCA2 molecules in the dimer of dimers observed by SAXS. Thus, this result supports that the ISCA2-ISCA2 interaction in the dimer of dimers is not a physiologically relevant species. On the other hand, the dimer of dimers formation might reflect a physiologically relevant tendency of ISCA2 to potentially dimerize with its physiological ISCA1 partner to form a ternary complex composed by [2Fe-2S] ISCA2-IBA57 and ISCA1. No data are, however, still available showing the formation of this ternary complex.

Our study provided a major contribution to understand the pathogenic role of a mutation on IBA57, Arg146Trp, among the twenty-five IBA57 mutations that have been up to now reported in multiple mitochondrial dysfunction syndrome $3^{24}$. Among them, seventeen are missense mutations and eight are truncating mutations ${ }^{24}$. Displaying all the missense residues on the IBA57 structure (Supplementary Fig. S8), it results that the majority of them (twelve out of seventeen) are part of the protein core with very low solvent accessibility (with relative solvent accessibility lower than 25\%) and thus not involved in protein-protein interactions. In vivo data, when available, showed that these pathogenic mutants have low IBA57 protein levels ${ }^{26-28}$. We can interpret this effect considering that the mutations of these residues largely affect protein stability as they are involved in intramolecular interactions, responsible of the correct fold of IBA57.Consequetnly, these mutations produces protein degradation in the cell. This effect was demonstrated for the first discovered pathogenic mutation of IBA57 gene ${ }^{27}$, i.e. Gln314 Pro, whose relative solvent accessibility is $23 \%$. On the contrary, it was found that the pathogenic mutation of Arg 146 to Trp does not significantly affect the stability of IBA57 in vivo ${ }^{22}$. The conserved residue Arg 146 is highly solvent accessible (Fig. 7) and its mutation clearly does not destabilize the protein overall structure. The Arg146Trp mutant displays, indeed, a 1D ${ }^{1} \mathrm{H}$ NMR spectrum typical of a folded protein and similar to that of the wild-type protein (Supplementary Fig. S7). The pathogenic impact of Arg mutation, being on the surface of the protein, originates from an impaired partner recognition. Indeed, Arg 146 is involved in an intermolecular electrostatic interaction with Asp/Glu of ISCA2 in our structural models and its mutation to Trp prevents complex formation. In conclusion, our structural data define the molecular grounds of the pathogenic impact of the aminoacid exchange Arg146Trp in IBA57, i.e. the function of IBA57 in mitochondrial [4Fe-4S] protein biogenesis is impaired by this mutation because of the abrogation of the [2Fe-2S] ISCA2-IBA57 complex formation. This result strongly claims on the functional relevance of the complex.

\section{Methods}

Protein production. IBA57 and ISCA2 proteins were obtained as previously reported ${ }^{18,21}$. The [2Fe-2S] ISCA2-IBA57 was obtained by chemically reconstituting with $\mathrm{FeCl}_{3}$ and $\mathrm{Na}_{2} \mathrm{~S}$ salts an apo ISCA2 and IBA57 mixture as previously reported ${ }^{21}$.

Mutagenesis and production of Arg146Trp pathogenic mutant. Arg146Trp mutant of human IBA57 was obtained through site-directed mutagenesis, using the pETDuet-1 vector containing the IBA57 gene as a template. The Arg146Trp mutant of human IBA57 was expressed and purified following the same protocol applied to the wild-type protein.

Analytical gel filtration and UV-Visible. Purified samples were loaded on a Superdex 75 HR 10/30 analytical column. Degassed $50 \mathrm{mM}$ phosphate buffer (pH 7.0), $150 \mathrm{mM} \mathrm{NaCl}$ and $5 \mathrm{mM}$ DTT was used as an eluent at a flow rate of $0.65 \mathrm{~mL} / \mathrm{min}$. UV-visible spectra were performed in degassed $50 \mathrm{mM}$ phosphate buffer at $\mathrm{pH} 7$, $5 \mathrm{mM}$ DTT and $150 \mathrm{mM} \mathrm{NaCl}$ on a Cary 50 Eclipse spectrophotometer.

SAXS data acquisition and analysis. Synchrotron SAXS data were collected at the EMBL beamline P12 $2^{49}$ at the PETRA III storage ring of DESY (Hamburg, Germany). The data on IBA57 and apo ISCA2 and were collected in on-line SEC-SAXS mode, while the data on the [2Fe-2S] ISCA2-IBA57 complex were collected using an automatic sample changer, flowing the sample during the exposure to avoid radiation damage. All samples were exposed in a flow capillary (quartz, $1.7 \mathrm{~mm}$ or $0.9 \mathrm{~mm}$ ). The parameters of both data collections are shown in Table 1. For SEC-SAXS, a buffer consisting of $50 \mathrm{mM}$ phosphate $\mathrm{pH} 7.0,150 \mathrm{mM} \mathrm{NaCl}, 5 \mathrm{mM}$ DTT and $3 \%$ (v/v) glycerol was used to separate the samples over a Superdex 200 Increase 10/300 column at a flow rate of $0.5 \mathrm{~mL} /$ $\mathrm{min}$ (IBA57) or $0.75 \mathrm{~mL} / \mathrm{min}$ (apo ISCA2). The SAXS curves of on IBA57 and apo ISCA2 were obtained from the main chromatographic peak. The [2Fe-2S] ISCA2-IBA57 complex, assembled with excess IBA57 (subsequently removed by analytical gel filtration) to favour complex formation, was measured in $50 \mathrm{mM}$ phosphate $\mathrm{pH} 7.0$, $150 \mathrm{mM} \mathrm{NaCl}, 5 \mathrm{mM}$ DTT at $\sim 1.8$ and $\sim 0.9 \mathrm{mg} / \mathrm{mL}$. As a (small) increase at low angles was observed for the more 
concentrated sample, likely due to some aggregation, subsequent data processing was performed using the dilute one. The scattered intensity was calibrated to absolute units using the scattering of water at $293 \mathrm{~K}$.

The primary data reduction was performed using SASFLOW ${ }^{50}$, initial data analysis with PRIMUS ${ }^{51}$, overall parameters were calculated using the ATSAS suite ${ }^{52}$. SEC-SAXS profiles were extracted from the chromatographic peak of interest using CHROMIXS ${ }^{53}$.

The SAXS curves from the atomic models were computed by CRYSOL ${ }^{54}$, rigid body modelling was performed using SASREF/SAREFCV ${ }^{55,56}$, multiphase $a b$ initio modelling, using MONSA ${ }^{57}$. The models were rendered with UCSF Chimera $^{58}$ and PyMOL Molecular Graphics System, Version $2.0^{59}$.

The SASREF modeling of apo ISCA2 was repeated 10 times in P2 symmetry; SASREF modeling of the complex employing the computationally generated [2Fe-2S] ISCA2-IBA57 heterodimers was repeated 20 times in P2 symmetry for both families $a$ and $b$ dimers.

The two-phase MONSA model was calculated by fitting simultaneously the SAXS curves of the ISCA2 homodimer and of the complex in the $s$-range of the data $\left[0.1,2.5 \mathrm{~nm}^{-1}\right]$. Here, one phase represented the IBA57 moiety, the other phase the ISCA2 moiety. The modeling was repeated 10 times in P2 symmetry.

Structural coordinates. Structural model of human IBA57 was already available in the Protein Data Bank (PDB ID 6QE3) $)^{21,33}$. An experimentally validated structure of monomeric apo ISCA2 was already available ${ }^{18}$. Structural models for human dimeric apo ISCA2 were generated with Modeller $9.20^{60}$ (https://salilab.org/modeller/) using the templates E. coli apo IscA (PDB ID 1R95 and 1S98) ${ }^{44,45}$, E. coli apo SufA (PDB ID 2D2A) ${ }^{61}$, while the structural model of monomeric [2Fe-2S] ISCA2 was generated with Modeller 9.20 using as template the T. elongates [2Fe-2S] IscA structure (PDB ID $1 \times 0 \mathrm{G})^{35}$. The [2Fe-2S] cluster bound to monomeric ISCA2 was included in the docking calculations by covalently linking one iron atom of the $[2 \mathrm{Fe}-2 \mathrm{~S}]$ cluster to the $\mathrm{S} \gamma$ atom of Cys 79 of ISCA2. [2Fe-2S] cluster was introduced with a total charge of +2 ; for the four cysteine ligands, the SH hydrogen was removed and the partial charges for side chain atoms were set to +0.18 for CB and -0.68 for SG. In this way, the total net charge of four cysteines plus the [2Fe-2S] cluster is zero.

Interface prediction. WHISCY program was used for interface predictions ${ }^{38}$. Multiple sequence alignments were constructed by first finding homologues of the human ISCA2 and IBA57 proteins using blastp against the $\mathrm{nr}$ database using default settings ${ }^{62}$, except that a restriction to only eukaryote sequences was used since the Fe-S cluster-delivery pathway might be different in prokaryotes as compared to eukaryotes. Subsequently, multiple sequence alignment was performed using ClustalW1.8 ${ }^{63}$. Interface predictions were used to generate AIRs. Specifically, active residues are defined based on interface predictions using WHISCY, passive residues are surface neighbors of active residues defined in the HADDOCK run (Supplementary Table S1).

Fe-S cluster-based restraints. We defined unambiguous interaction restraints between the iron atoms and the $\mathrm{S} \gamma$ atoms of the coordinating cysteines on both ISCA2 and IBA57. The restraint distance was $2.3 \AA$; residues involved in those restraints are listed in Supplementary Table S1.

Docking protocol. Structural models of the [2Fe-2S] ISCA2-IBA57 complex were calculated using the protein-protein docking program HADDOCK 2.2 (high ambiguity driven protein-protein docking) by following the standard HADDOCK procedure ${ }^{36,37}$. HADDOCK uses AIRs defined from any available information about the interface. In our case, active residues were based on interface prediction by WHISCY, and included as AIRs in the docking calculations as mentioned above. In addition to these AIRs, experimentally based unambiguous interaction restraints (Fe-S cluster-based restraints) deriving from previous mutagenesis data ${ }^{21}$ were included. The final ensemble of 200 solutions was ranked based on the HADDOCK score with standard weights ${ }^{64}$. The water-refined models were clustered based on the default fraction of common contacts, FCC $=0.75$, with the minimum number of elements in a cluster of $4^{39}$. NACCESS is the program used to calculate the atomic and residue accessibilities from a PDB format file. The models were rendered with MOLMOL program ${ }^{65}$.

\section{Data availability}

The authors confirm that the docking data are available from the corresponding author on request, and the SAXS models and the corresponding data are being submitted to the SASBDB, with accession codes SASDGD2 (IBA57), SASDGE2 (ISCA2), SASDGF2 (complex).

Received: 9 September 2019; Accepted: 11 November 2019;

Published online: 12 December 2019

\section{References}

1. Beinert, H. Iron-sulfur proteins: ancient structures, still full of surprises. J Biol. Inorg. Chem 5, 2-15 (2000).

2. Beinert, H., Holm, R. H. \& Munck, E. Iron-sulfur clusters: nature's modular, multipurpose structures. Science 277, 653-659 (1997).

3. Johnson, D. C., Dean, D. R., Smith, A. D. \& Johnson, M. K. Structure, function, and formation of biological iron-sulfur clusters. Annu. Rev. Biochem 74, 247-281 (2005).

4. Lill, R. Function and biogenesis of iron-sulphur proteins. Nature 460, 831-838 (2009).

5. Ye, H. \& Rouault, T. A. Human iron-sulfur cluster assembly, cellular iron homeostasis, and disease. Biochemistry 49, 4945-4956 (2010).

6. Ciofi-Baffoni, S., Nasta, V. \& Banci, L. Protein networks in the maturation of human iron-sulfur proteins. Metallomics 10, 49-72 (2018).

7. Stehling, O., Wilbrecht, C. \& Lill, R. Mitochondrial iron-sulfur protein biogenesis and human disease. Biochimie 100, 61-77 (2014).

8. Maio, N. \& Rouault, T. A. Iron-sulfur cluster biogenesis in mammalian cells: New insights into the molecular mechanisms of cluster delivery. Biochim. Biophys. Acta 1853, 1493-1512 (2015).

9. Boniecki, M. T., Freibert, S. A., Muhlenhoff, U., Lill, R. \& Cygler, M. Structure and functional dynamics of the mitochondrial Fe/S cluster synthesis complex. Nature communications 8, 1287 (2017). 
10. Mapolelo, D. T., Zhang, B., Naik, S. G., Huynh, B. H. \& Johnson, M. K. Spectroscopic and functional characterization of iron-bound forms of Azotobacter vinelandii (Nif)IscA. Biochemistry 51, 8056-8070 (2012).

11. Muhlenhoff, U., Richter, N., Pines, O., Pierik, A. J. \& Lill, R. Specialized function of yeast Isa1 and Isa2 proteins in the maturation of mitochondrial [4Fe-4S] proteins. J. Biol. Chem 286, 41205-41216 (2011).

12. Johansson, C. et al. The crystal structure of human GLRX5: iron-sulfur cluster co-ordination, tetrameric assembly and monomer activity. Biochem. J 433, 303-311 (2011).

13. Banci, L. et al. [2Fe-2S] cluster transfer in iron-sulfur protein biogenesis. Proc. Natl. Acad. Sci. USA 111, 6203-6208 (2014).

14. Sheftel, A. D. et al. The human mitochondrial ISCA1, ISCA2, and IBA57 proteins are required for [4Fe-4S] protein maturation. Mol. Biol. Cell 23, 1157-1166 (2012).

15. Ye, H. et al. Glutaredoxin 5 deficiency causes sideroblastic anemia by specifically impairing heme biosynthesis and depleting cytosolic iron in human erythroblasts. J. Clin. Invest 120, 1749-1761 (2010).

16. Gelling, C., Dawes, I. W., Richhardt, N., Lill, R. \& Muhlenhoff, U. Mitochondrial Iba57p is required for Fe/S cluster formation on aconitase and activation of radical SAM enzymes. Mol Cell Biol 28, 1851-1861 (2008).

17. Uzarska, M. A., Dutkiewicz, R., Freibert, S. A., Lill, R. \& Muhlenhoff, U. The mitochondrial Hsp70 chaperone Ssq1 facilitates Fe/S cluster transfer from Isu1 to Grx5 by complex formation. Mol. Biol. Cell 24, 1830-1841 (2013).

18. Brancaccio, D. et al. Formation of [4Fe-4S] clusters in the mitochondrial iron-sulfur cluster assembly machinery. J. Am. Chem Soc 136, 16240-16250 (2014).

19. Brancaccio, D. et al. [4Fe-4S] Cluster Assembly in Mitochondria and Its Impairment by Copper. J. Am. Chem. Soc 139, 719-730 (2017).

20. Beilschmidt, L. K. et al. ISCA1 is essential for mitochondrial Fe4S4 biogenesis in vivo. Nature communications 8, 15124 (2017).

21. Gourdoupis, S., Nasta, V., Calderone, V., Ciofi-Baffoni, S. \& Banci, L. IBA57 Recruits ISCA2 to Form a [2Fe-2S] Cluster-Mediated Complex. J Am Chem Soc 140, 14401-14412 (2018).

22. Debray, F. G. et al. Mutation of the iron-sulfur cluster assembly gene IBA57 causes fatal infantile leukodystrophy. J. Inherit. Metab Dis 38, 1147-1153 (2015)

23. Liu, M. et al. Phenotypic spectrum of mutations in IBA57, a candidate gene for cavitating leukoencephalopathy. Clin Genet 93, 235-241 (2018).

24. Hamanaka, K. et al. Expanding the phenotype of IBA57 mutations: related leukodystrophy can remain asymptomatic. J Hum Genet 63, 1223-1229 (2018).

25. Lossos, A. et al. Fe/S protein assembly gene IBA57 mutation causes hereditary spastic paraplegia. Neurology 84, 659-667 (2015)

26. Torraco, A. et al. Novel mutations in IBA57 are associated with leukodystrophy and variable clinical phenotypes. Journal of neurology 264, 102-111 (2017).

27. Ajit, B. N. et al. Mutation of the iron-sulfur cluster assembly gene IBA57 causes severe myopathy and encephalopathy. Hum. Mol. Genet 22, 2590-2602 (2013).

28. Ishiyama, A. et al. IBA57 mutations abrogate iron-sulfur cluster assembly leading to cavitating leukoencephalopathy. Neurology. Genetics 3, e184 (2017)

29. Alfadhel, M. Multiple Mitochondrial Dysfunctions Syndrome 4 Due to ISCA2 Gene Defects: A Review. Child neurology open 6 (2019).

30. Al-Hassnan, Z. N. \& Kaya, N. In GeneReviews (eds M. P. Adam et al.) (University of Washington, Seattle. 1993)

31. Alaimo, J. T. et al. Loss-of-function mutations in ISCA2 disrupt $4 \mathrm{Fe}-4 \mathrm{~S}$ cluster machinery and cause a fatal leukodystrophy with hyperglycinemia and mtDNA depletion. Hum Mutat 39, 537-549 (2018).

32. Lebigot, E. et al. Impact of mutations within the [Fe-S] cluster or the lipoic acid biosynthesis pathways on mitochondrial protein expression profiles in fibroblasts from patients. Mol Genet Metab 122, 85-94 (2017).

33. Gourdoupis, S., Nasta, V., Ciofi-Baffoni, S., Banci, L. \& Calderone, V. In-house high-energy-remote SAD phasing using the magic triangle: how to tackle the P1 low symmetry using multiple orientations of the same crystal of human IBA57 to increase the multiplicity. Acta Crystallogr D Struct Biol 75, 317-324 (2019).

34. Banci, L. et al. Modeling protein-protein complexes involved in the cytochrome $c$ oxidase copper-delivery pathway. J. Proteome Res 6, 1530-1539 (2007).

35. Morimoto, K. et al. The asymmetric IscA homodimer with an exposed [2Fe-2S] cluster suggests the structural basis of the Fe-S cluster biosynthetic scaffold. J. Mol. Biol 360, 117-132 (2006).

36. Dominguez, C., Boelens, R. \& Bonvin, A. M. HADDOCK: a protein-protein docking approach based on biochemical or biophysical information. J. Am. Chem. Soc 125, 1731-1737 (2003).

37. van Zundert, G. C. et al. The HADDOCK2.2 Web Server: User-Friendly Integrative Modeling of Biomolecular Complexes. J. Mol. Biol 428, 720-725 (2016).

38. de Vries, S. J., van Dijk, A. D. \& Bonvin, A. M. WHISCY: what information does surface conservation yield? Application to datadriven docking. Proteins 63, 479-489 (2006).

39. Rodrigues, J. P. et al. Clustering biomolecular complexes by residue contacts similarity. Proteins 80, 1810-1817 (2012).

40. Banci, L. et al. Copper(I)-mediated protein-protein interactions result from suboptimal interaction surfaces. Biochem. J 422, 37-42 (2009).

41. Banci, L., Bertini, I., McGreevy, K. S. \& Rosato, A. Molecular recognition in copper trafficking. Nat. Prod. Rep 27, 695-710 (2010).

42. Al-Hassnan, Z. N. et al. ISCA2 mutation causes infantile neurodegenerative mitochondrial disorder. J Med Genet 52, 186-194 (2015).

43. Toldo, I. et al. Neonatal mitochondrial leukoencephalopathy with brain and spinal involvement and high lactate: expanding the phenotype of ISCA2 gene mutations. Metabolic brain disease 33, 805-812 (2018).

44. Bilder, P. W., Ding, H. \& Newcomer, M. E. Crystal structure of the ancient, Fe-S scaffold IscA reveals a novel protein fold. Biochemistry 43, 133-139 (2004).

45. Cupp-Vickery, J. R., Silberg, J. J., Ta, D. T. \& Vickery, L. E. Crystal structure of IscA, an iron-sulfur cluster assembly protein from Escherichia coli. J. Mol. Biol 338, 127-137 (2004).

46. Beilschmidt, L. K. \& Puccio, H. M. Mammalian Fe-S cluster biogenesis and its implication in disease. Biochimie 100, 48-60 (2014).

47. Waller, J. C. et al. A role for tetrahydrofolates in the metabolism of iron-sulfur clusters in all domains of life. Proc. Natl. Acad. Sci. USA 107, 10412-10417 (2010).

48. Chen, J. W., Sun, C. M., Sheng, W. L., Wang, Y. C. \& Syu, W. J. Expression Analysis of Up-Regulated Genes Responding to Plumbagin in Escherichia coli. J Bacteriol 188, 456-463 (2006).

49. Blanchet, C. E. et al. Versatile sample environments and automation for biological solution X-ray scattering experiments at the P12 beamline (PETRA III, DESY). J Appl Crystallogr 48, 431-443 (2015).

50. Franke, D., Kikhney, A. G. \& Svergun, D. I. Automated acquisition and analysis of small angle X-ray scattering data. Nucl Instrum Meth A 689, 52-59 (2012).

51. Petoukhov, M. V. et al. New developments in the ATSAS program package for small-angle scattering data analysis. Journal Of Applied Crystallography 45, 342-350 (2012).

52. Franke, D. et al. ATSAS 2.8: a comprehensive data analysis suite for small-angle scattering from macromolecular solutions. J Appl Crystallogr 50, 1212-1225 (2017). 
53. Panjkovich, A. \& Svergun, D. I. CHROMIXS: automatic and interactive analysis of chromatography-coupled small-angle X-ray scattering data. Bioinformatics 34, 1944-1946 (2018).

54. Svergun, D. I., Barberato, C. \& Koch, M. H. J. CRYSOL - a program to evaluate X-ray solution scattering of biological macromolecules from atomic coordinates. J. Appl. Crystallogr 28, 768-773 (1995).

55. Petoukhov, M. V. \& Svergun, D. I. Global Rigid Body Modeling of Macromolecular Complexes against Small-Angle Scattering Data. Biophys J 89, 1237-1250 (2005).

56. Petoukhov, M. V. \& Svergun, D. I. Joint use of small-angle X-ray and neutron scattering to study biological macromolecules in solution. Eur Biophys J 35, 567-576 (2006).

57. Svergun, D. I. Restoring low resolution structure of biological macromolecules from solution scattering using simulated annealing. Biophys J 76, 2879-2886 (1999).

58. Pettersen, E. F. et al. UCSF Chimera - A Visualization System for Exploratory Research and Analysis. J Comp Chem 25, 1605-1612 (2004).

59. The PyMOL Molecular Graphics System v. Version 1.8 (2015).

60. Sali, A. \& Blundell, T. L. Comparative protein modelling by satisfaction of spatial restraints. J. Mol. Biol 234, 779-815 (1993).

61. Wada, K. et al. Crystal structure of Escherichia coli SufA involved in biosynthesis of iron-sulfur clusters: implications for a functional dimer. FEBS Lett 579, 6543-6548 (2005)

62. Altschul, S. F. et al. Gapped BLAST and PSI-BLAST: a new generation of protein database search programs. Nucleic Acids Res 25, 3389-3402 (1997).

63. Thompson, J. D., Higgins, D. G. \& Gibson, T. J. CLUSTAL W: improving the sensitivity of progressive multiple sequence alignment through sequence weighting, position-specific gap penalties and weight matrix choice. Nucleic Acids Res 22, 4673-4680 (1994).

64. van Dijk, A. D., Kaptein, R., Boelens, R. \& Bonvin, A. M. Combining NMR relaxation with chemical shift perturbation data to drive protein-protein docking. J Biomol NMR 34, 237-244 (2006).

65. Koradi, R., Billeter, M. \& Wüthrich, K. MOLMOL: a program for display and analysis of macromolecular structure. J. Mol. Graphics 14, 51-55 (1996).

66. Hajizadeh, N. R., Franke, D., Jeffries, C. M. \& Svergun, D. I. Consensus Bayesian assessment of protein molecular mass from solution X-ray scattering data. Sci Rep 8, 7204 (2018).

67. Kozin, M. B. \& Svergun, D. I. Automated matching of high-and low-resolution structural models. Journal of applied crystallography. Journal Of Applied Crystallography 34, 33-41 (2001).

\section{Acknowledgements}

S.D.V. and D.S. acknowledge funding from the Joachim Herz Stiftung, Hamburg, Germany (Infectophysics grant). We thank the SPC facility at EMBL Hamburg for technical support and C. Borges, M. Gräwert, A. Gruzinov, C. Jeffries for helpful interactions during the SAXS measurements performed for this work. L.B. acknowledge funding from iNEXT, grant number 653706 , and from timb ${ }^{3}$, grant number 810856 , both funded by the Horizon 2020 programme of the European Commission. The authors acknowledge the support and the use of resources of Instruct-ERIC, a Landmark ESFRI Research Infrastructure, and specifically CERM/CIRMMP Italy Centre. This article is based upon work from COST Action CA15133, supported by the European Cooperation in Science and Technology (COST). Dr. Andrea Giachetti and Davide Sala are warmly acknowledged for helping us in running HADDOCK calculations.

\section{Author contributions}

Conceptualization, S.C.B. and L.B.; Investigation, V.N., S.D.V. and S.G.; Formal Analysis, S.D.V. and D.S.; WritingOriginal Draft, S.C.B. and S.D.V.; Writing-Review \& Editing, L.B., D.S., S.C.B. and S.D.V.; Visualization, V.N., S.C.B. and S.D.V.; Funding Acquisition, L.B.; Supervision, D.S. and L.B.

\section{Competing interests}

The authors declare no competing interests.

\section{Additional information}

Supplementary information is available for this paper at https://doi.org/10.1038/s41598-019-55313-5.

Correspondence and requests for materials should be addressed to L.B.

Reprints and permissions information is available at www.nature.com/reprints.

Publisher's note Springer Nature remains neutral with regard to jurisdictional claims in published maps and institutional affiliations.

(c) (i) Open Access This article is licensed under a Creative Commons Attribution 4.0 International License, which permits use, sharing, adaptation, distribution and reproduction in any medium or format, as long as you give appropriate credit to the original author(s) and the source, provide a link to the Creative Commons license, and indicate if changes were made. The images or other third party material in this article are included in the article's Creative Commons license, unless indicated otherwise in a credit line to the material. If material is not included in the article's Creative Commons license and your intended use is not permitted by statutory regulation or exceeds the permitted use, you will need to obtain permission directly from the copyright holder. To view a copy of this license, visit http://creativecommons.org/licenses/by/4.0/.

(C) The Author(s) 2019 